\title{
TARP FILTERING OF BLOCK-TRANSFORM COEFFICIENTS FOR EMBEDDED IMAGE CODING
}

\author{
Vijay P. Shah, James E. Fowler, and Nicholas H. Younan \\ Department of Electrical and Computer Engineering \\ GeoResources Institute, Mississippi State ERC \\ Mississippi State University, Mississippi State, MS
}

\begin{abstract}
Tarp filtering, an image coder with a simple implementation, is coupled with a block-based discrete cosine transform equipped with pre- and postfiltering. The prefilter reduces intra-block and inter-block correlation of the block-based coefficients, resulting in coefficients that are less correlated and thereby more suitable to tarp filtering. Experimental results show that the proposed coder achieves a significant improvement in rate-distortion performance as compared to the corresponding tarp coder in its original wavelet-based formulation for images with highly detailed content. A similar gain over JPEG2000 is seen for these same images, while, for images that are mostly smooth, the proposed coder performs comparably to JPEG2000.
\end{abstract}

\section{INTRODUCTION}

Image coders in current widespread use generally employ either a discrete wavelet transform (DWT) or a more traditional blockbased transform such as the discrete cosine transform (DCT). The success of recent wavelet-based coders is due to advanced context modeling, exploitation of zero clustering and parent-child relationships, and adaptive entropy coding with sophisticated context conditioning. The recent JPEG2000 standard [1], generally considered to be state of the art in wavelet-based image coding, employs many of these techniques and, as a result, entails a rather complicated implementation. In order to reduce implementation complexity, there has been an interest in alternative, less complicated coders. One such coder is tarp filtering $[2,3]$.

Tarp filtering, which does not use sophisticated context conditioning like many other wavelet-based coders, features quick, online adaption for the estimation of probability densities in regions with low activity. However, the fact that the tarp filter is isotropic in nature limits its ability to capture edge and pattern information [4]. This leads to relatively inferior performance as compared to context-based methods when significant correlation exists between neighboring pixels. It has been shown that tarp filtering performs well on images consisting of smooth features, yet is outperformed by JPEG2000 and other coders for certain images with high detail content (e.g., "barbara"), suggesting that sophisticated context-based modeling is more efficient than tarp filtering for those types of images. However, as we show in this paper, one can improve the performance of tarp filtering for highly detailed images by replacing the wavelet transform originally employed in [2-4] with a block-based transform coupled with a prefilter and postfilter.

Block-based image coders partition an image into blocks, performing a transform (usually the DCT) on each block individually.
However, some correlation remains along the block boundaries, resulting in blocking and ringing artifacts in the reconstructed image [5]. In order to improve the performance of the block-based transform, we adopt the prefilter and postfilter proposed by Tran et al. $[5,6]$. This invertible prefiltering is applied along the block boundaries before performing the block transform in order to reduce intra-block and inter-block correlation. The prefilter acts as a smoothing operator on the image but does not remove any highfrequency components from the block-it simply shifts them to block boundaries. Since the block is smooth, greater energy compaction is obtained. The postfilter is an inverse filter which acts as a smoothing operator at the block boundaries, which, in turn, removes the notorious blocking artifacts often seen in block-based image compression.

To deploy tarp filtering on prefiltered, block-based DCT coefficients, we reorder the block-transform coefficients into a waveletlike pyramid structure. Such coefficient reordering schemes [710] have been used in the past in order to deploy onto blocktransform coefficients the embedded quantization and highly conditioned entropy coding such as used in SPIHT and other waveletbased coders. In our approach, coefficient reordering permits the application of tarp filtering onto block-based DCT coefficients. The encoder of the proposed system is depicted in Fig. 1, while the system is described below.

\section{TARP FILTERING}

Simard et al. proposed tarp filtering for wavelet-based image coding in $[2,3]$. Tarp filtering consists of coding a binary-valued field by applying a first-order recursive filter to estimate the probability of the next symbol being a 1 , and then driving a nonadaptive arithmetic coder with the estimated probability. Tarp filtering uses three 1D filters where each filter runs in a different direction-the first runs from left to right, the second runs from right to left (after processing a full row), and the third runs from top to bottom for each column. The 1D filters, in effect, efficiently implement a 2D convolution, which, in turn, embodies a 2D Parzen windows probability estimate; a parameter $\alpha$, called the learning rate, controls the spread of the Parzen window. As originally described in [2], each bitplane of the quantized wavelet coefficients is encoded independently using the tarp-filter coder, thereby making the bitstream non-embedded. An embedded tarp coder was implemented in QccPack [11] by adopting successive-approximation bitplane coding, using tarp filtering to generate probability estimates for the coding of the coefficient significance states. However, when used in this embedded sense, tarp filtering typically yields ratedistortion performance somewhat inferior to that of JPEG2000.

Tian et al. [4] observed that, because of the strict raster-scan 
processing of tarp filtering, the resulting bitstream is not typically arranged in a maximally embedded order. To improve performance, three-pass fractional-bitplane coding was coupled with coefficient classification to produce the embedded tarp filtering with classification (TCE) coder [4]. TCE performs three passes for each bitplane-the first pass encodes bits of non-zero neighbor coefficients using an adaptive arithmetic coder, the second pass estimates the probability of zero-run coefficients using tarp filtering and a nonadaptive arithmetic coder, while the final pass encodes refinement bits using adaptive arithmetic coding. The resulting TCE coder offers embedding coding with rate-distortion performance comparable to that of JPEG2000 [4].

\section{PREFILTERING, POSTFILTERING, AND COEFFICIENT REARRANGEMENT}

In this section, we replace the wavelet transform of the tarp-filter coders of [2-4] with a block-based DCT, using prefiltering to reduce intra-block and inter-block correlations. Subsequent experimental results demonstrate that this transform modification improves the rate-distortion performance of tarp filtering for images of high detail content.

\subsection{Prefiltering and Postfiltering}

Tran et al. [5,6] proposed prefiltering in a separable fashion at block boundaries to reduce the correlation between adjacent blocks for traditional block-based transforms. The image is reconstructed in an overlapping manner using a corresponding postfilter that is the exact inverse of the prefilter, and it, too, is applied in a separable fashion. The prefilter, $\mathbf{P}$, is

$$
\mathbf{P}=\frac{1}{2}\left[\begin{array}{cc}
\mathbf{I} & \mathbf{J} \\
\mathbf{J} & -\mathbf{I}
\end{array}\right]\left[\begin{array}{cc}
\mathbf{I} & \mathbf{0} \\
\mathbf{0} & \mathbf{V}
\end{array}\right]\left[\begin{array}{cc}
\mathbf{I} & \mathbf{J} \\
\mathbf{J} & -\mathbf{I}
\end{array}\right],
$$

where $\mathbf{I}, \mathbf{J}$, and $\mathbf{0}$ are the $\frac{M}{2} \times \frac{M}{2}$ identity matrix, the anti-diagonal matrix, and the null matrix, respectively, with $M$ being the filter size. The controlling parameter of the prefilter matrix is matrix $\mathbf{V}$,

$$
\mathbf{V}=\mathbf{J} \mathbf{C}_{M / 2}^{\mathrm{II}} \mathbf{S C}_{M / 2}^{\mathrm{IV}} \mathbf{J}
$$

where $\mathbf{C}_{M / 2}^{\mathrm{II}}$ and $\mathbf{C}_{M / 2}^{\mathrm{IV}}$ are the $\frac{M}{2}$-point type-II and type-IV DCT matrices, respectively. Biorthogonality is introduced via diagonal matrix $\mathbf{S}=\operatorname{diag}\{s, 1,1, \ldots, 1\}$, which, in turn, controls the amount of smoothness provided within the block, as well as the energy compaction. The choice of parameter $s$ is important, and a few values suggested are $\frac{8}{5}, \frac{3}{2}, \frac{25}{16}$, the golden ratio $\frac{1+\sqrt{5}}{2}$, or the ratio of any two consecutive numbers of the Fibonacci series $[5,6]$. Here, we also investigate the use of another ratio obtained from the Lucas series, $\{2,1,3,4,7,11,18, \ldots\}$, which has properties similar to the Fibonacci series. From this series, additional values for parameter $s$, namely, $3, \frac{4}{3}$, and $\frac{7}{4}$, are available; we have found experimentally that $s=\frac{4}{3}$ offers consistently strong performance.

\subsection{Coefficient Rearrangement}

In order to perform tarp-based embedded coding, or TCE-based fractional-bitplane coding, the coefficients from the block-based DCT are rearranged into a pyramidal structure akin to the dyadic pyramid typically produced by wavelet transforms. Fig. 2 depicts the rearrangement of an array of $8 \times 8$ DCT coefficients to form a pyramid structure similar to a three-level dyadic wavelet pyramid [7-10]. Fig. 3 depicts the rearranged DCT coefficients of "lenna" using a block size of $8 \times 8$.

\section{RESULTS}

Throughout the results presented in this section, DCT-TCE denotes the coder proposed above using a block-based DCT coupled with the TCE algorithm of [4], while DCT-Tarp refers to a block-based DCT used with the embedded tarp filtering from the tarp coder of [11]. Correspondingly, the wavelet-based coders are called DWT-TCE and DWT-Tarp. The coders are tested on images from the the USC database. ${ }^{1}$ All images are 8-bit grayscale images of size $512 \times 512$, except "chemical plant," which is $256 \times 256$, and "airport," which is $1024 \times 1024$. Kakadu ${ }^{2}$ Version 4.4 is used to produce JPEG2000 results. All wavelet transforms use a 5-level decomposition with the popular 9/7 biorthogonal filters. For all the experiments, the learning rate for DWT-TCE and DWT-Tarp is set to $\alpha=0.4$ and $\alpha=0.6$, respectively, as suggested in [2,4]. In order to determine $\alpha$ for DCT-TCE and DCT-Tarp, $\alpha$ is varied on the interval $[0.3,0.7]$. Results indicate that both DCT-TCE and DCT-Tarp work well for all $\alpha \in[0.3,0.6]$; thus, $\alpha$ is set to the same values as used by the wavelet-based counterparts. Experiments are conducted using block sizes of $8 \times 8,16 \times 16$, and $32 \times 32$ for the block-based DCT.

Rate-distortion performance for DCT-TCE, JPEG2000, and DWT-TCE for several images is shown in Figs. 4-6. It is evident that the DCT-based coders provide rate-distortion performance superior to that of their wavelet-based counterparts, particularly for the images that contain more significant detail features-precisely those images for which DWT-Tarp does not perform as well as JPEG2000. For example, the average performance gain of DCTTCE for "barbara" is about $1.4 \mathrm{~dB}$ over JPEG2000 and $1.7 \mathrm{~dB}$ over DWT-TCE. Similarly, for "elaine" and "chemical plant," the average increase in performance is greater than $0.7 \mathrm{~dB}$. For images that consist mostly of "smooth" features (e.g., "lenna"), DCT-TCE performs comparably to both DWT-TCE and JPEG2000; the average difference in performance between the various coders for such smooth images is on the order of only $0.2 \mathrm{~dB}$. Table 1 presents the PSNR performance of the various coders at a fixed rate of 0.5 bits per pixel (bpp). Table 1 indicates that DCT-TCE provides superior rate-distortion performance for highly detailed images, while, for images with "smooth features," it offers performance roughly comparable to that of DWT-TCE and JPEG2000. Also evident is that a block size of $16 \times 16$ outperforms a block size of $8 \times 8$.

\section{CONCLUSIONS}

In this paper, we proposed coupling the tarp-filtering TCE coder of [4] with a block-based DCT equipped with the pre- and postfiltering of $[5,6]$. In the resulting DCT-TCE coder, the prefiltering of the image tends to reduce intra-block and inter-block correlation of the block-based DCT coefficients, resulting in coefficients that are less correlated and thereby well-suited to tarp filtering. Experimental results showed that the proposed DCT-TCE coder achieves a significant improvement in rate-distortion performance as compared to the corresponding wavelet-based tarp coder, DWT-TCE

\footnotetext{
${ }^{1}$ http://sipi.usc.edu/database

${ }^{2}$ http: //www.kakadusoftware.com
} 


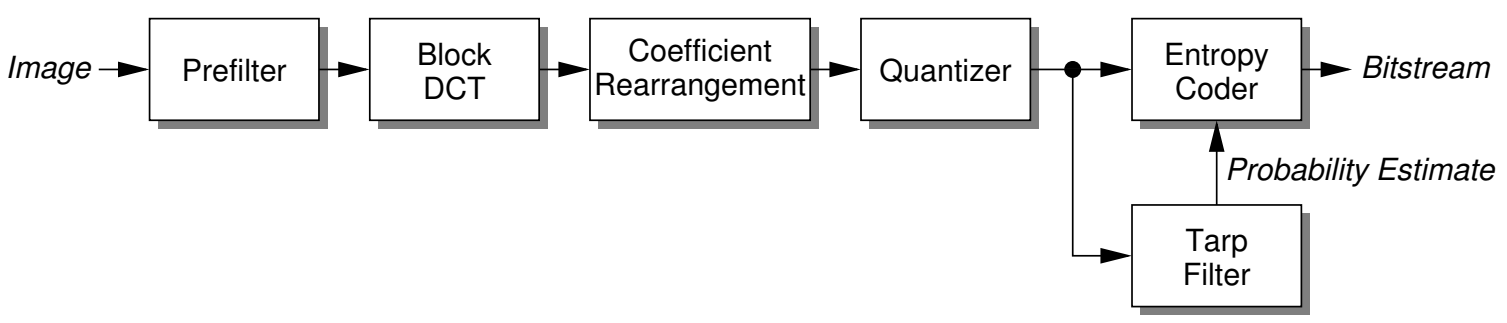

Figure 1: Encoder of the proposed system.

Table 1: PSNR (dB) at rate of $0.5 \mathrm{bpp}$.

\begin{tabular}{|c|c|c|c|c|c|c|c|c|c|c|c|}
\hline \multirow[b]{3}{*}{ Images } & \multirow{2}{*}{\multicolumn{3}{|c|}{ 9/7 Wavelet }} & \multicolumn{4}{|c|}{ DCT } & \multicolumn{4}{|c|}{ DCT with pre/postfilter } \\
\hline & & & & \multicolumn{2}{|c|}{$8 \times 8$} & \multicolumn{2}{|c|}{$16 \times 16$} & \multicolumn{2}{|c|}{$8 \times 8$} & \multicolumn{2}{|c|}{$16 \times 16$} \\
\hline & JP2K & TCE & Tarp & TCE & Tarp & TCE & Tarp & TCE & Tarp & TCE & Tarp \\
\hline barbara & 32.2 & 31.8 & 31.1 & 30.9 & 30.7 & 32.3 & 31.9 & 32.9 & 32.6 & 33.8 & 33.2 \\
\hline elaine & 33.5 & 33.6 & 33.5 & 33.2 & 33.1 & 33.7 & 33.6 & 33.7 & 33.7 & 34.5 & 34.4 \\
\hline lenna & 37.3 & 37.3 & 36.7 & 36.0 & 35.8 & 36.6 & 36.2 & 36.9 & 36.6 & 37.1 & 36.7 \\
\hline goldhill & 33.2 & 33.2 & 33.0 & 32.4 & 32.1 & 32.9 & 32.6 & 33.1 & 32.8 & 33.2 & 33.0 \\
\hline fishing boat & 33.3 & 33.3 & 32.9 & 32.3 & 31.9 & 32.6 & 32.2 & 33.0 & 32.7 & 33.0 & 32.6 \\
\hline tank & 34.6 & 34.7 & 34.6 & 34.0 & 33.9 & 34.4 & 34.2 & 34.6 & 34.5 & 34.7 & 34.5 \\
\hline boat bridge & 27.2 & 27.2 & 27.1 & 26.6 & 26.5 & 26.9 & 26.8 & 27.1 & 27.0 & 27.1 & 27.0 \\
\hline baboon & 25.5 & 25.7 & 25.5 & 25.1 & 24.9 & 25.5 & 25.1 & 25.6 & 25.3 & 25.7 & 25.3 \\
\hline texture mosaic & 18.0 & 18.0 & 17.8 & 18.1 & 18.0 & 18.6 & 18.5 & 18.3 & 18.1 & 18.5 & 18.2 \\
\hline chemical plant & 28.9 & 29.4 & 29.1 & 28.8 & 28.5 & 29.3 & 29.1 & 29.5 & 29.3 & 29.7 & 29.5 \\
\hline airport & 30.4 & 30.4 & 30.1 & 30.0 & 29.7 & 30.2 & 29.9 & 30.4 & 30.1 & 30.3 & 30.1 \\
\hline Average & 30.4 & 30.4 & 30.1 & 29.8 & 29.5 & 30.3 & 30.0 & 30.5 & 30.3 & 30.7 & 30.4 \\
\hline
\end{tabular}

\begin{tabular}{|c|c|cc|cccc|}
\hline 0 & 1 & 4 & 5 & 16 & 17 & 20 & 21 \\
\cline { 1 - 2 } 2 & 3 & 6 & 7 & 18 & 19 & 22 & 23 \\
\cline { 1 - 2 } 8 & 9 & 12 & 13 & 24 & 25 & 28 & 29 \\
10 & 11 & 14 & 15 & 26 & 27 & 30 & 31 \\
\hline 32 & 33 & 36 & 37 & 48 & 49 & 52 & 53 \\
34 & 35 & 38 & 39 & 50 & 51 & 54 & 55 \\
40 & 41 & 44 & 45 & 56 & 57 & 60 & 61 \\
42 & 43 & 46 & 47 & 58 & 59 & 62 & 63 \\
\hline
\end{tabular}

Figure 2: Pyramidal form for rearranged DCT coefficients of an $8 \times 8$ DCT (adapted from [7]).

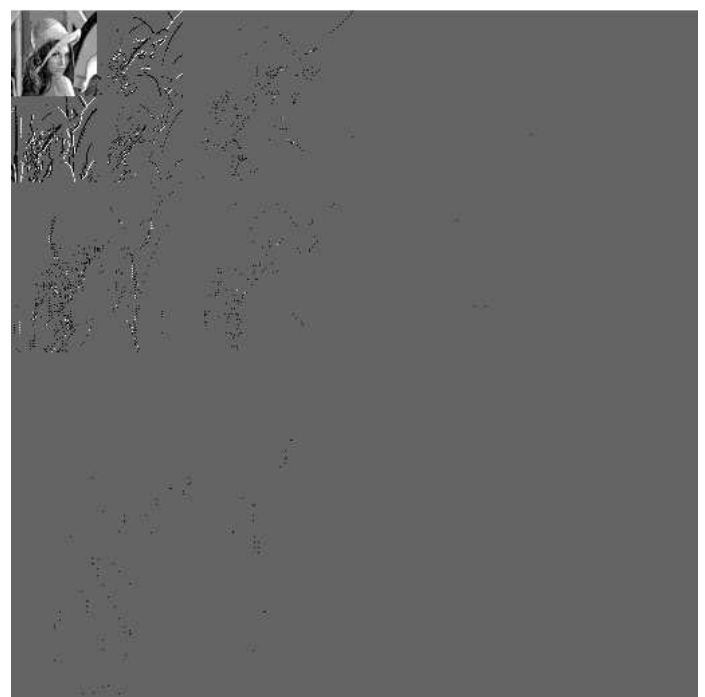

Figure 3: Rearranged coefficients of "lenna" using a DCT block size of $8 \times 8$. 


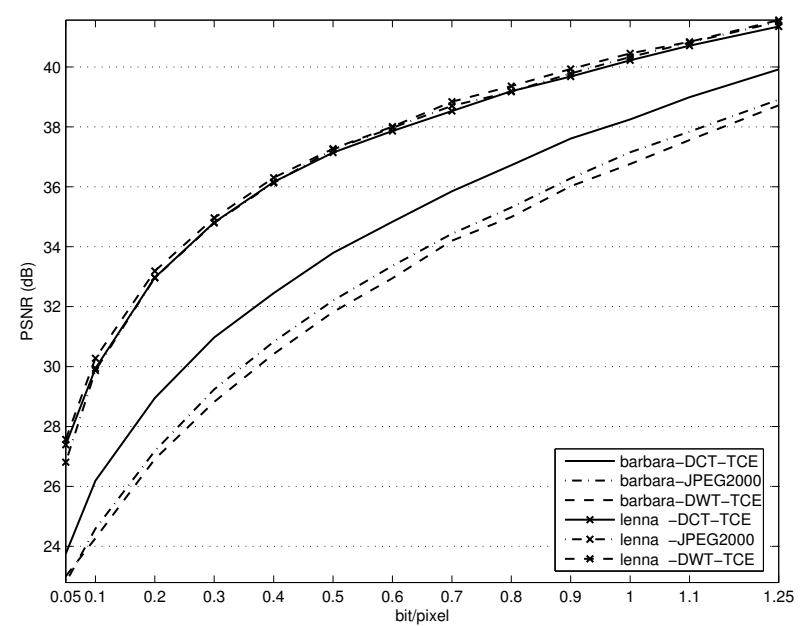

Figure 4: Rate-distortion performance for "barbara" and "lenna." DCT block size $=16 \times 16$.

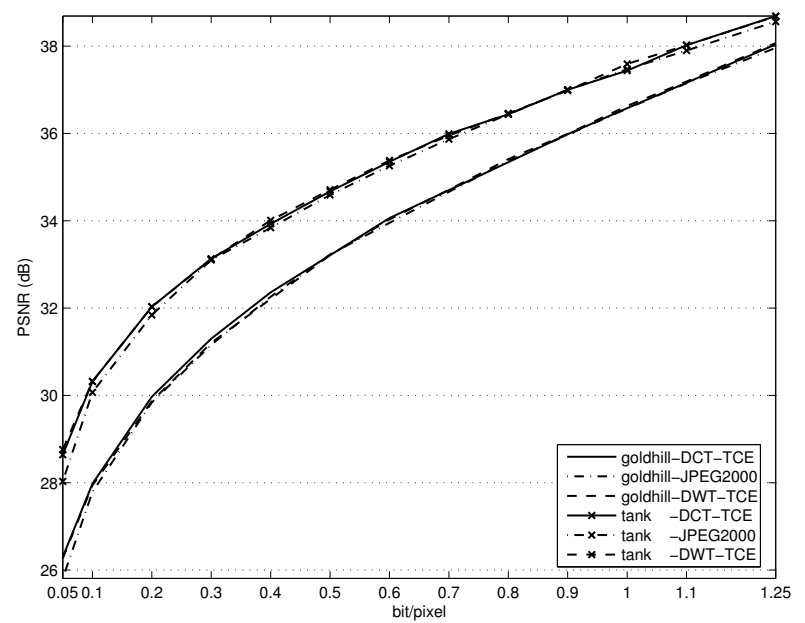

Figure 5: Rate-distortion performance for "goldhill" and "tank." DCT block size $=16 \times 16$.

[4], for images with highly detailed content. A similar gain over JPEG2000 was seen for those same images. On the other hand, for images that are mostly smooth, the proposed DCT-TCE performed comparably to DWT-TCE and JPEG2000.

\section{ACKNOWLEDGMENT}

The authors thank J. Rucker for providing help with the experimental results.

\section{REFERENCES}

[1] Information Technology-JPEG 2000 Image Coding System-Part 1: Core Coding System, ISO/IEC 15444-1, 2000.

[2] P. Simard, D. Steinkraus, and H. Malvar, "On-line adaptation in image coding with a 2-D tarp filter," in Proceedings of the IEEE Data Compression Conference, J. A. Storer and M. Cohn, Eds., Snowbird, UT, April 2002, pp. 23-32.

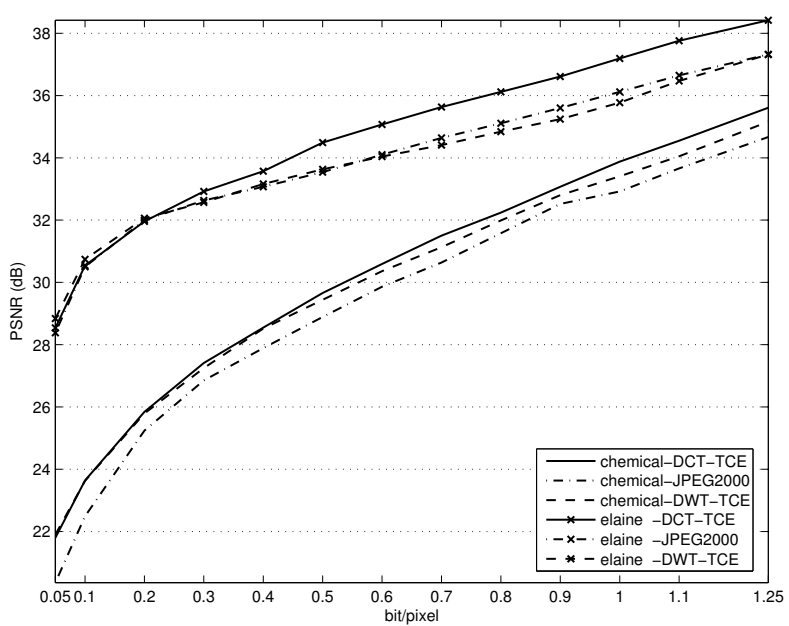

Figure 6: Rate-distortion performance for "elaine" and "chemical plant." DCT block size $=16 \times 16$.

[3] P. Y. Simard, C. J. C. Burges, D. Steinkraus, and H. S. Malvar, "Image compression with on-line and off-line learning," in Proceedings of the International Conference on Image Processing, vol. 2, Barcelona, Spain, September 2003, pp. 259-262.

[4] C. Tian and S. S. Hemami, "An embedded image coding system based on tarp filter with classification," in Proceedings of the International Conference on Acoustics, Speech, and Signal Processing, vol. 3, Montreal, Canada, May 2004, pp. 49-52.

[5] T. D. Tran, J. Liang, and C. Tu, "Lapped transform via timedomain pre- and post-filtering," IEEE Transactions on Signal Processing, vol. 51, no. 6, pp. 1557-1571, June 2003.

[6] C. Tu and T. D. Tran, "Context-based entropy coding of block transform coefficients for image compression," IEEE Transactions on Image Processing, vol. 11, no. 11, pp. 12711283, November 2002.

[7] Z. Xiong, O. G. Guleryuz, and M. T. Orchard, "A DCTbased embedded image coder," IEEE Signal Processing Letters, vol. 3, no. 11, pp. 289-290, November 1996.

[8] T. D. Tran and T. Q. Nguyen, "A progressive transmission image coder using linear phase uniform filterbanks a block transforms," IEEE Transactions on Image Processing, vol. 8, no. 11, pp. 1493-1507, November 1999.

[9] H. S. Malvar, "Fast progressive image coding without wavelets," in Proceedings of the IEEE Data Compression Conference, J. A. Storer and M. Cohn, Eds., Snowbird,UT, March 2000, pp. 243-252.

[10] Y.-A. Jeong and C.-K. Cheong, "A DCT-based embedded image coder using wavelet structure of DCT for very low bit rate video codec," IEEE Transactions on Consumer Electronics, vol. 44, no. 3, pp. 500-508, August 1998.

[11] J. E. Fowler, "QccPack: An open-source software library for quantization, compression, and coding," in Applications of Digital Image Processing XXIII, A. G. Tescher, Ed. San Diego, CA: Proc. SPIE 4115, August 2000, pp. 294-301. 